\title{
Common Risk Factor Approach for Oral Diseases- A SWOT Analysis
}

\author{
Sreekanth Bose* \\ Department of Public Health Dentistry, India \\ *Corresponding Author: Sreekanth Bose, Department of Public Health Dentistry, India.
}

Received: July 05, 2019; Published: August 08, 2019

DOI: 10.31080/ASDS.2019.03.0611

\begin{abstract}
Non Communicable Diseases (NCD) are on a rising trend in developed and developing world. Oral diseases are now considered as public health problem due to its effect on quality of life. Both these diseases are having many epidemiological similarities. Classical method of disease prevention and control strategies are not applicable there. Due to the presence of similar determinants in disease formation, a new method called- common risk factor approach is devised as a preventive strategy for them. The method aims to control risk factors which are common to NCDs and oral diseases.

The method is based on principles of health promotion and is cost effective, but at the same time it has a many limitations too. The method have limited evidence regarding its application, hence it should be practiced with caution.
\end{abstract}

Keywords: Risk Factor; Oral Diseases; NCDs

\section{Introduction}

Of 56.9 million global deaths in $2016,40.5$ million, or $71 \%$, were due to noncommunicable diseases (NCDs). The four main NCDs are cardiovascular diseases, cancers, diabetes and chronic lung diseases. The burden of these diseases is rising disproportionately among lower income countries and populations. In 2016, over three quarters of NCD deaths -- 31.5 million -- occurred in lowand middle-income countries with about $46 \%$ of deaths occurring before the age of 70 in these countries [1]. Oral diseases, including dental caries, periodontal disease and oral cancer, are neglected but important NCDs with a significant burden on overall health [2]. There is a need for a single strategy to prevent and manage noncommunicable diseases [3].

The Multiple Risk Factor Intervention Trial emphasized the limitations of the lifestyle approach wherein Health professionals have traditionally focused upon changing the behaviours of their patients for promoting health and preventing disease, but failed to understand that there were problems related to social and cultural milieu rather than of the individual. This paved in the path for Common Risk Factor Approach revolutionising the concept that Oral health problems have risk factor in common with a number of important chronic diseases, and it's inefficient to target each disease separately when they have similar origins [4].

A number of chronic diseases such as heart disease, cancer strokes, injuries and oral diseases have risk factors in common and many risk factors are relevant to more than one chronic disease. Such risk factor oriented strategies are more rational than those directed at specific diseases [4]. The key concept underlying the integrated common risk approach is that promoting general health by controlling a small number of risk factors may have a major impact on a large number of diseases at a lower cost, greater efficiency and effectiveness than disease specific approaches [5].

\section{Rise of NCDs}

For centuries, communicable diseases were the main causes of death around the world. Life expectancy was often limited by uncontrolled epidemics. After the second World War, with medical research achievements in terms of vaccination, antibiotics and improvement of life conditions, non communicable diseases (NCDs) started causing major problems in industrialized countries. Heart diseases, cancer, diabetes, chronic pulmonary and mental diseases became a real burden for health systems in developed countries. For a while, these diseases were associated with economic development and so called diseases of the rich. Then, by the dawn of the third millennium, NCDs appeared sweeping the entire globe, with an increasing trend in developing countries where, the transition imposes more constraints to deal with the double burden of infective and non-infective diseases in a poor environment characterized by ill-health systems [6].

Eighty percent of NCD related deaths occur in low- and middle-income countries, especially as these countries undergo 
socioeconomic improvement after reductions in infectious disease. The World Health Organization predicts a global increase of $17 \%$ in NCDs over the next decade [7].

\section{Oral disease burden}

Oral diseases such as dental caries, periodontal disease, tooth loss, oral mucosal lesions and oropharyngeal cancers, human immunodeficiency virus/acquired immunodeficiency syndrome (HIV/AIDS)- related oral disease and orodental trauma are major public health problems worldwide. Poor oral health may have a profound effect on general health, and several oral diseases are related to chronic diseases (e.g. diabetes). The experience of pain, problems with eating, chewing, smiling and communication due to missing, discoloured or damaged teeth have a major impact on people's daily lives and well-being. Furthermore, oral diseases restrict activities at school, at work and at home causing millions of school and work hours to be lost each year throughout the world [8].

Dental caries and periodontal disease have historically been considered the most important global oral health burdens. Worldwide, the prevalence of dental caries among adults is high as the disease affects nearly $100 \%$ of the population in the majority of countries. Tooth loss in adult life may also be attributable to poor periodontal health. Severe periodontitis, which may result in tooth loss, is found in 5-20\% of most adult populations worldwide $[9,10]$. Oropharyngeal cancer is more common in developing than developed countries. The prevalence of oral cancer is particularly high among men and it is the eighth most common cancer worldwide [11]. Studies have demonstrated the negative impact on oral health of HIV infection [12]. Approximately $40-50 \%$ of people who are HIV-positive have oral disease caused by fungal, bacterial or viral infections that often occur early in the course of the disease $[13,14]$.

\section{NCDs and Oral diseases-Epidemiological similarities}

Oral diseases are non communicable and multifactorial in origin which share modifiable risk factors with the leading NCDs, including tobacco use, harmful alcohol consumption, and unhealthy diets. Both are the diseases of modern civilization and mediated by lifestyle transitions. Biomedical model of disease causation is not applicable and traditional interventions like vaccines or pharmacotherapy is of less significance in them. Risk factor modification is the best preventive strategy for both oral diseases and NCDs [14].

\section{Common risk factors}

Oral health and systemic health are closely related. This may be due to the fact that severe oral diseases and non-communicable chronic diseases have common risk factors such as tobacco use, diet, excessive alcohol consumption, stress, and poor hygiene practices. In addition, bidirectional interrelationships between oral and general diseases have been demonstrated, for example, in the case of periodontal and systemic diseases [2]
- Tobacco: The use of tobacco has been implicated in a large number of diseases. Smokers more often develop cancers of the lung, mouth, throat, pancreas, kidney, and urinary tract and have coronary heart disease and stroke, respiratory diseases, diabetes, and ulcers than nonsmokers. Smokers also have a high risk of periodontal disease and lesions of the oral mucosa [16].

- Diet: Diet is a risk factor for many NCDs. Diebetes Mellitus, Cardiovascular diseases, cancers and dental caries are associated with unhealthy diet and consumption of sugar. Sugar is called as white tobacco and has significant adverse effect on health.

- Alcohol: High alcohol consumption increases the risk of a wide variety of conditions such as increased blood pressure, liver cirrhosis, cardiovascular disease, diabetes, and cancers of the mouth. Recent research also indicates that excessive alcohol consumption is associated with increased severity of periodontal disease [17].

- Stress: It is well known that cardiovascular disease, diabetes, and other chronic diseases are related to psychosocial factors, but there is also evidence that stress is linked to periodontal disease [18].

\section{SWOT analysis}

\section{Strength}

- $\quad$ The method is based on the multifactorial theory of causation.

- Focus on control of risk factors

- Long term benefits are expected

- $\quad$ Cost effective

- Uses the principles of health promotion (Community participation, Integration of programs, Focus on prevention)

- $\quad$ Reduce social inequalities

- Applicable in developing and developed countries.

\section{Opportunity}

Threat

- Integration of services and programs (Inclusion of oral health into NHM)

- Horizontal programs

- Incorporation of health promoting concepts into national policies (eg:Taxation for sugar)

- Activities to achieve Sustainable Development Goals

\section{Weakness}

- Scarcity of evidence in practical application.

- $\quad$ Limited to certain diseases specific determinants (eg; Oral hygiene for oral diseases) which have more attributable risk

- Practical difficulties in controlling risk factors which has deep rooted social implications (Diet, Alcohol, Tobacco)
- Fail to address disease

Table 


\section{Conclusion}

Common risk factor approach is a rational method advocated by World Health Organisation to prevent and control Noncommunicable diseases. Oral diseases and NCDs have epidemiological similarities, and the preventive modalities can be integrated based on common risk factors. The most important risk factors which need immediate attention are diet, tobacco, alcohol and stress.

The common risk factor approach is based on principles of health promotion and is cost effective. However the evidence regarding applicability of this method is limited and it fail to address risk factors specific to diseases.

The common risk factor approach should be practiced with caution. Future research is needed to generate appropriate evidence regarding this method.

\section{Bibliography}

1. NCD mortality and morbidity. Global Health Observatory (GHO) data. World Health Organization.

2. Petersen PE. "The World Oral Health Report 2003: continuous improvement of oral health in the 21st century- -the approach of the WHO Global Oral Health Programme". Community Dent Oral Epidemiology. 31.1 (2003): 3-23.

3. Yale Shweta. "Common risk factor approach: Finding common ground for better health outcomes". International Journal of Contemporary Medical Research 4.6 (2017): 1367-1370.

4. Sheiham A and Watt RG. "The common risk factor approach: a rational basis for promoting oral health". Community Dent Oral Epidemiology 28.6 (2000): 399-406.

5. Grabauskas V. "Integrated programme for community health in noncommunicable disease (Interhealth). In: Leparski E, editor. The prevention of non-communicable diseases: experiences and prospects. Copenhagen: WHO Regional Office for Europe; (1987): 285-310.

6. Boutayeb A and Boutayeb S. "The burden of non communicable diseases in developing countries". International journal for equity in health 4.1 (2005): 2 .

7. Alwan A. "WHO action plan for the global strategy for the prevention and control of noncommunicable diseases". Geneva, Switzerland: World Health Organization, 2008.

8. Petersen PE., et al. "The global burden of oral diseases and risks to oral health". Bulletin of the World Health Organization 83.9 (2005): 661-669.

9. Global oral health data bank. Geneva: World Health Organization; 2004

10. WHO oral health country/area profile. Geneva: World Health Organization.
11. Stewart BW and Kleihues P. "World cancer report". Lyon: International Agency for Research on Cancer (2003).

12. Coogan MM and Sweet SP. "Oral manifestations of HIV in the developing and developed world". Oral Diseases 82 (2002): 5-190.

13. Greenspan JS and Greenspan D. "The epidemiology of the oral lesions of HIV infection in the developed world". Oral Diseases 8.2 (2002): 34-39.

14. Holms HK and Stephen LXG. "Oral lesions of HIV infection in developing countries”. Oral Diseases 8.2 (2002): 40-43.

15. Integrating Oral Health with NonCommunicable Diseases as an Essential Component of General Health: WHO's Strategic Orientation for the African Region

16. Petersen PE and Ogawa H. "Strengthening the prevention of periodontal disease: the WHO approach". Journal of periodontology. 76.12 (2005): 2187-2193.

17. Tezal M., et al. "The effect of alcohol consumption on periodontal disease". Journal of Periodontology 72.2 (2001): 183-189.

18. Genco RJ., et al. "Relationship of stress, distress, and inadequate coping behaviors to periodontal disease". Journal of Periodontology 70.7 (1999): 711-723.

Volume 3 Issue 9 September 2019 (C) All rights are reserved by Sreekanth Bose. 